\title{
Simulation of novel cell-like topological structures with quantum walk
}

\author{
S. Panahiyan ${ }^{1,2,3, a}{ }_{\mathbb{D}}$, S. Fritzsche $e^{1,2,3, b}$ \\ ${ }^{1}$ Helmholtz-Institut Jena, Fröbelstieg 3, 07743 Jena, Germany \\ 2 GSI Helmholtzzentrum für Schwerionenforschung, 64291 Darmstadt, Germany \\ 3 Theoretisch-Physikalisches Institut, Friedrich-Schiller-University Jena, 07743 Jena, Germany
}

Received: 11 July 2020 / Accepted: 28 July 2020 / Published online: 5 August 2020

(C) The Author(s) 2020

\begin{abstract}
We demonstrate how quantum walk can simulate exotic cell-like structures for topological phases and boundary states. These cell-like structures contain the three known boundary states of Dirac cone, Fermi arc and flat bands alongside of all trivial and non-trivial phases of BDI family of topological phases. We also characterize the behavior of boundary states through Bloch spheres. In addition, we investigate the topological phase transitions and critical behavior of the system that take place over boundary states through curvature function. We confirm that critical behavior of the simulated topological phenomena can be described by peak-divergence scenario. We extract the critical exponents and length scale, establish a scaling law and show that band crossing is 1 . Furthermore, we find the correlation function through Wannier states and show that it decays as a function of length scale.
\end{abstract}

Topological phases are symmetry-preserving phases of matter that are described by global topological orders (topological invariants) [1]. Due to these global orders, topological phases exhibit exotic properties including integer Hall effect [1], fractional charges and magnetic monopoles [2] and existence of topological insulators [3,6]. The topological phenomena first were reported in condensed-matter materials such as topological insulators and superconductors [3-7], and later, they were observed in cold atoms in optical lattices [8,9], phononic states in mechanical oscillators [10] and photons in quantum walks [11-18]. Specially, the topological phenomena observed in quantum walks have attracted a lot of attentions. This is because the controllability over walker's behavior in quantum walk makes them versatile simulators of topological phenomena which can be used to suppress limits on the dynamics of strongly driven systems and observe new features such as the robust edge states [11].

Quantum walks are universal frameworks [19] developed in quantum information and computation to simulate other quantum systems and phenomena [20,21]. The quantum walk is the result of driving its protocol. Generally, this protocol consists of coin and shift operators which are applied on a state of a walker (i.e., a fermion or a photon) repeatedly. The coin operators modify the internal state of the walker (i.e., spin or polarization), while the shift operators change walker's external state (i.e., position or orbital angular momentum) based on internal states. So far, the quantum walks were utilized to realize all known kinds of topological phases in one and two dimensions [22-26] and some of three-dimensional ones

\footnotetext{
a e-mail: shahram.panahiyan@uni-jena.de (corresponding author)

b e-mail: s.fritzsche@gsi.de
} 
[27] extract topological invariants [28] and investigate bulk-boundary correspondence [29,30] and topological phase transitions $[31,32]$.

In previous study [33], we confirmed that by utilizing step-dependent coins [34] in splitstep quantum walk, we can simulate exotic cell-like structure for topological phenomena [33]. In this paper, we mainly focus on these cell-like structures and address the following issues. First of all, we determine the types of phases and boundary states that are present in each cell. We confirm that each cell contains two non-trivial phases with different topological invariants and two trivial ones alongside of two Fermi arc, two flat band and one Dirac cone boundary states. In addition, we use Bloch spheres to specify the differences in behavior of different boundary states. Lastly, we investigate the critical behavior of the simulated topological phenomena through curvature function. We extract critical exponent and length scale and establish that band crossing is one and a scaling law is valid. Additionally, we show that characteristic behavior of the curvature and correlation functions highly depends on the type of the boundary state and the energy of the gap-closer.

T. Kitagawa et al showed that by changing the protocol of the quantum walk from simplestep (shift-coin operation) into split-step (shift-coin-shift-coin operation), one can further enrich the phase space and simulates all possible phases in one and two dimensions [22]. Here, we use this modification in the protocol of the walk as well. The walker has one external degree of freedom (one-dimensional position space) with two internal states. The split-step protocol of the quantum walk with step-dependent coin is

$$
\widehat{U}=\widehat{S}_{\uparrow} \widehat{C}_{\alpha} \widehat{S}_{\downarrow} \widehat{C}_{\beta},
$$

which indicates that one step of quantum walk comprises rotation of internal states with $\widehat{C}_{\beta}$, displacement of its position with $\widehat{S}_{\downarrow}$, a second rotation of internal states with $\widehat{C}_{\alpha}$ and finally, its displacement with $\widehat{S}_{\uparrow}$. The step-dependent coins are $\widehat{C}_{\beta}=e^{-\frac{i T \beta}{2} \sigma_{y}}$ and $\widehat{C}_{\alpha}=e^{-\frac{i T \alpha}{2} \sigma_{y}}$ with $\sigma_{y}$ being Pauli matrix, $T$ number of the step and $\alpha$ and $\beta$ rotation angles. The shift operators are in diagonalized forms of $\widehat{S}_{\uparrow}=e^{\frac{i k}{2}\left(\sigma_{z}-1\right)}$ and $\widehat{S}_{\downarrow}=e^{\frac{i k}{2}\left(\sigma_{z}+1\right)}$ in which we have used discrete Fourier transformation $\left(|k\rangle=\sum_{x} e^{-\frac{i k x}{2}}|x\rangle\right)$.

Since the quantum walk is done by successive application of its protocol, it is possible to map the unitary evolution of its protocol to a stroboscopic evolution under an effective Hamiltonian given by

$$
\widehat{H}(k)=i \ln \widehat{U}(k)=E(k) \boldsymbol{n}(k) \cdot \boldsymbol{\sigma},
$$

where $E(k)$ is the (quasi-) energy dispersion, $\boldsymbol{\sigma}$ is the Pauli matrices, and $\boldsymbol{n}(k)$ defines the coin eigenstates at each (quasi-) momentum $k$. The Hamiltonian has periodicity of $H(k+2 \pi)=$ $H(k)$ which enables us to limit momentum to first Brillouin zone, $[-\pi, \pi]$. The protocol of quantum walk (1) is Hermitian, and its determinant is 1 . Consequently, $\widehat{H}(k)$ is traceless which results in the symmetry of $E(k)=E(-k)$ and $E(k)$ being limited to $[-\pi, \pi]$.

There are two internal states; therefore, there are two bands of energy which we obtain as

$$
E(k)= \pm \cos ^{-1}\left(\kappa_{\alpha} \kappa_{\beta} \cos (k)-\lambda_{\alpha} \lambda_{\beta}\right),
$$

in which $\cos \left(\frac{T j}{2}\right)=\kappa_{j}$ and $\sin \left(\frac{T j}{2}\right)=\lambda_{j}$ where $j$ could be $\alpha, \beta$. The energy bands are gapped, and the gap between them could close. The topological phases are characterized by gapped bands of energy. In contrast, the boundary states are recognized by gapless energy bands. Next, we find $\boldsymbol{n}(k)$ in form of

$$
\boldsymbol{n}(k)=\frac{\zeta}{|\zeta|}
$$


in which $\zeta=\left(\kappa_{\alpha} \lambda_{\beta} \sin (k), \lambda_{\alpha} \kappa_{\beta}+\kappa_{\alpha} \lambda_{\beta} \sin (k),-\kappa_{\alpha} \kappa_{\beta} \sin (k)\right)$.

The Hamiltonian has particle-hole $(\widehat{\mathcal{P}} \equiv \widehat{K}$ with $\widehat{K}$ being the complex conjugation operator), chiral $\left(\widehat{\Gamma}=\kappa_{\beta} \sigma_{x}+\lambda_{\beta} \sigma_{z}\right)$ and time-reversal $(\widehat{\mathcal{T}} \equiv \widehat{\Gamma} \widehat{\mathcal{P}})$ symmetries with the symmetries square to +1 . Therefore, the protocol of the quantum walk (1) can simulate BDI family of topological phases in one dimension. Additionally, due to these symmetries, $\boldsymbol{n}(k)$ is constrained to lie on a great circle of the Bloch sphere and winding number becomes topological invariant. The winding number is specified by number of times $\boldsymbol{n}(k)$ winds around the origin as $k$ varies in the first Brillouin zone. Since our Hamiltonian is Hermitian, the winding number is integer in which zero corresponds to a trivial topological phase, while nonzero indicates non-trivial topological phases $[37,38]$. The winding number is calculated by

$$
w=\int_{-\pi}^{\pi}\left[\boldsymbol{n}(k) \times \partial_{k} \boldsymbol{n}(k)\right] \cdot \boldsymbol{A} \frac{d k}{2 \pi},
$$

in which $\boldsymbol{A}=\left(\kappa_{\beta}, 0, \lambda_{\beta}\right)$.

To fully characterize the simulated topological phenomena by quantum walk, we obtain the group velocity as $V(k)=\partial_{k} E(k)= \pm\left|n_{z}\right|$ which has indication that the value of group velocity spans $[-1,1]$. b) group velocity becomes ill-defined if energy bands close their gap.

Overall, we can set down the following guidelines for distinguishing different simulated topological phenomena; topological phases are characterized by gapped energy bands with well-defined $\boldsymbol{n}(k)$, winding number and group velocity. In contrast, the boundary states have gapless energy bands and ill-defined $\boldsymbol{n}(k)$, winding number and group velocity. The type of a topological phase is determined by its winding number. On the other hand, different types of boundary states are categorized based on how the energy bands close their gap. Generally, we have three types of the boundary states known as Dirac cone (linear dispersive behavior), Fermi arc (nonlinear dispersive behavior) and flat bands (dispersionless behavior) [35]

To investigate simulable edge states by the protocol of the quantum walk (1), we replace the second rotation angle, $\alpha$ with a position-dependent one [22]

$$
\alpha=\frac{1}{2}\left(\alpha_{1}+\alpha_{2}\right)+\frac{1}{2}\left(\alpha_{1}-\alpha_{2}\right) \tanh (x / 3) .
$$

The resultant protocol has all three symmetries of particle-hole, time-reversal and chiral with the symmetries square to +1 . Therefore, we can simulate BDI family of the topological phases. The new protocol provides a spatially inhomogeneous quantum walk. For our study, we consider $\alpha_{2}=-\alpha_{1}$. Using this scheme, we can create boundaries which has distinctive topological phases on each side of it. In such cases, a single localized state with energy $E=0$ or $E= \pm \pi$ will reside at the boundary. Therefore, we have an edge state with energy $E=0$ or $E= \pm \pi$.

Generally, the topological invariant $C$ for one-dimensional systems can be calculated using [39]

$$
C=\int_{-\pi}^{\pi} F(k, M) \frac{d k}{2 \pi},
$$

where $F(k, M)$ is referred to as the curvature function and $M$ is a certain parameter determined by the system under investigation. The topological phase transition is defined as a certain jump in topological invariant as $M$ tunes up to a critical point $M_{c}$. The curvature function is an even function $\left(F\left(k_{0}+\delta k, M\right)=F\left(k_{0}-\delta k, M\right)\right)$ around the point where energy gap closes. The topological invariant $C$ remains fixed in a topological phase in parameter space of $M$, while the profile of $F(k, M)$ modifies. This property is the key ingredient to investigate topological phase transitions, critical exponent, etc., for topological phenomena. 
The critical behavior of the curvature functions falls into two categories [39]: a) Peakdivergence scenario in which the curvature function peaks at the gapless point. This is done by the peak narrowing and increasing its height as the system approaches the critical point $(M \rightarrow$ $M_{c}$ ) and eventually the peak diverges and flips sign across the transition. b) Shell-divergence scenario in which the extremum of the curvature function forms a $d$-1-dimensional shell around gapless point. As $M \rightarrow M_{c}$, the radius of the shell reduces and extremum gradually diverges and flips the sign across transition point. The first critical behavior happens for linear band crossing of $m=1$, while the second case takes place for higher order of band crossing.

For BDI family of topological phases, the crossing band is $m=2 \mathbb{Z}+1$ [39]. Comparing (7) with (5), we find the curvature function as

$$
F(k, M)=\left[\boldsymbol{n}(k) \times \partial_{k} \boldsymbol{n}(k)\right] \cdot \boldsymbol{A},
$$

and the $M$ is parameterized by rotation angles of the coin operators and step number of the walk.

In our simulation of topological phases, we consider one of the rotation angles to be linearly related to each other through $\beta=(\alpha+\pi) / 3$. The existence of step-dependent coin introduces dynamicality as a feature for simulated topological phenomena by our quantum walk. This means at each step, the number of the topological phases, boundary states, edge states, their types and their places could be controlled by step number of the walk. We focus on two specific step of the walk: 6 th and 8 th steps.

In simulation, cell-like structures for topological phases and boundary states emerge (see Fig. 1a). Each cell is characterized by two flat bands boundary states that act as cell's walls. Inside these walls, there are two Fermi arcs with a Dirac cone between them. Each cell contains two non-trivial phases with winding number +1 and -1 , and two trivial phases (see Fig. 1c). Therefore, each cell contains all three topological phases of the BDI family in addition to all three types of boundary states available for one-dimensional systems.

The phase structure at each side of boundary states uniquely depends on type of the boundary state. The neighboring topological phases of flat bands boundary states are nontrivial ones with the same winding number. In contrast, the Dirac cone boundary states reside at the border of two trivial phases. Only the Fermi arcs boundary states separate two distinctive topological phases from one another. Therefore, the topological phase transitions taking place at flat bands and Dirac cones are between two identical phases, whereas at Fermi arcs, the type of topological phase changes. It should be noted though that two non-trivial phases with different winding numbers cannot be in neighboring of each other.

The behavior of the $\boldsymbol{n}(k)$ as $k$ traverses the first Brillouin zone differs for each type of boundary state (see Fig. 1e). For flat bands, the $\boldsymbol{n}(k)$ would reside only in the origin of the Bloch sphere. If the boundary state is Dirac cone type, $\boldsymbol{n}(k)$ will span the diameter of the sphere. Finally, for Fermi arc-type boundary states, $\boldsymbol{n}(k)$ passes the origin and lies on half of the greatest circle on Bloch sphere.

In case of inhomogeneous quantum walk (position-dependent rotation angle), the boundary states could host edge states with $E=0$ and $\pm \pi$. If we fix the position and vary the rotation angle through $[-\pi, \pi]$, we observe the formation of the cell-like structure for edge states and topological phases (see Fig. 1b). The cells are identical to previously discussed ones with the same structure for topological phases, boundary (edge) states and their types (see Fig. 1d). The only differences lie in the fact that locations (the value of rotation angle) of boundary (edge) states are modified. It is also possible to invoke bulk-boundary correspondence for picturing the edge states at the interfaces between two phases using guidelines provided in Ref. [24]. To do so, we use a set of numbers $Q_{0}$ and $Q_{\pi}$. We choose a point in parameter space of phase $A$, connect it to a point in phase $B$ and count the number of times 
E

(a)

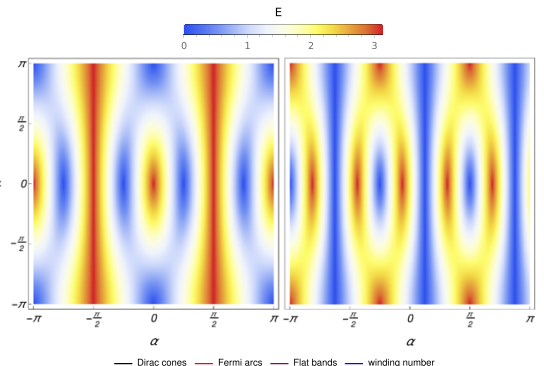

(c)

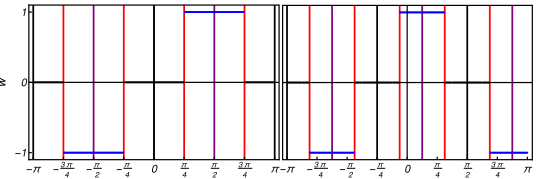

(b)

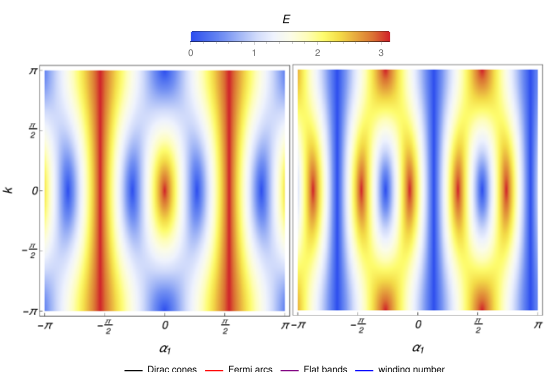

(d)

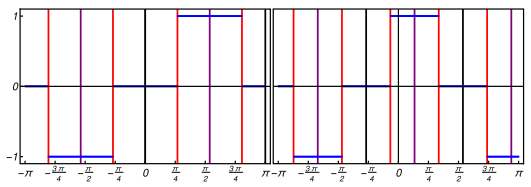

(e)

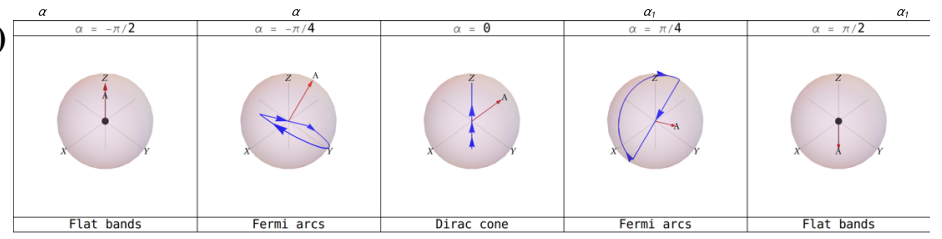

Fig. 1 In $\mathbf{a}$ and $\mathbf{b}$, energy as a function of momentum and rotation angle $\alpha(\beta=(\alpha+\pi) / 3)$ for steps six (left panels) and eight (right panels) is plotted. In a, we consider the rotation angle to be position-independent. We recognize cell-like structure in energy diagrams by two gapless flat bands (walls of cell). The interior of this cell contains one gapless Dirac cone located between two gapless Fermi arcs. In b, we consider rotation angle to be position-dependent $\left(\alpha=\alpha_{1} \tanh (x / 3)\right)$ with $x=5$. We observe that cell-like structure appears here as well for boundary (edge) states. In $\mathbf{c}$ and $\mathbf{d}$, the winding number (topological invariant) and boundary (edge) states for two cases of position-independent $\mathbf{c}$ and position-dependent $\mathbf{d}$ rotation angle $\alpha$ are plotted. We observe that each cell contains two non-trivial phases with different winding numbers and two trivial ones. Only for Fermi arc boundary (edge) states, two distinct topological phases reside at neighboring of each other. In contrast, the phases around flat bands and Dirac cone boundary states are identical. The topological phases around flat bands are non-trivial ones, while for Dirac cones, they are trivial ones. In $\mathbf{e}$, we have the Bloch sphere presentation of $\boldsymbol{n}(k)$ (blue curves) as $k$ traverses the first Brillouin zone in a plane orthogonal to $\boldsymbol{A}$ for 6 th step with $\beta=(\alpha+\pi) / 3$. The characteristic behavior of $\boldsymbol{n}(k)$ significantly differs for each boundary states. For flat bands, $\boldsymbol{n}(k)$ resides only at the origin, while in Dirac cone case, it spans the diameter of the sphere. In case of Fermi arcs, it forms a closed loop passing the origin and over half of the great circle on the sphere

that the gap around $E=0$ and $E= \pm \pi$ closes to obtain $Q_{0}$ and $Q_{\pi}$. Using the results, we can recognize differences in both invariants of $Q_{0}$ and $Q_{\pi}$ for gapped phases in different regions of $\alpha_{1}$.

In general, we can find the curvature function for these quantum walks as

$$
F(k, \alpha)=\frac{-\cos (k) \lambda_{2 \alpha} \kappa_{\beta}-2 \kappa_{\alpha}^{2} \lambda_{\beta}}{2 \sin ^{2}(k) \kappa_{\alpha}^{2}+2\left(\cos (k) \kappa_{\alpha} \lambda_{\beta}+\lambda_{\alpha} \kappa_{\beta}\right)^{2}} .
$$

For Fermi arcs and Dirac cones, the energy bands close their gaps at $k=k_{c}=0$ and $k=k_{c}= \pm \pi$. This indicates that to investigate the limiting behavior of the curvature function, we should set $k=0$ or $k= \pm \pi$. We consider the $k=0$ with $\beta=(\alpha+\pi) / 3$ which leads to followings for critical rotation angles

$$
\lim _{\alpha \rightarrow 0^{+}} F\left(k_{c}, \alpha\right)=\lim _{\alpha \rightarrow \frac{\pi}{4}^{+}} F\left(k_{c}, \alpha\right)=-\infty,
$$


(a)

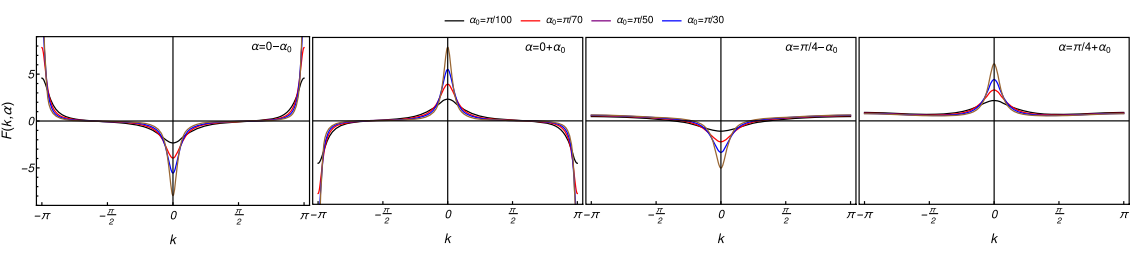

(b)

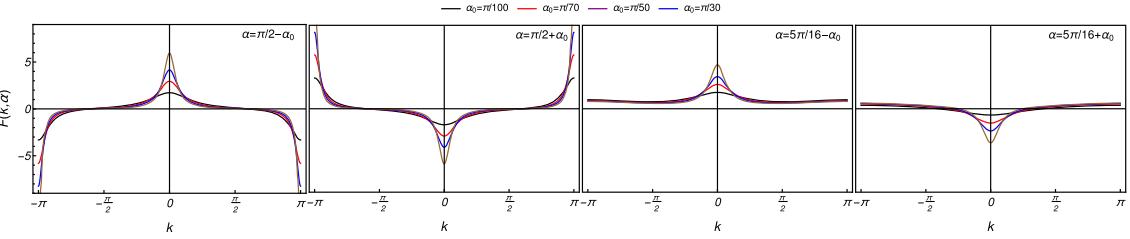

Fig. 2 Curvature function as a function of momentum for different rotation angle of $\alpha$ with $\beta=(\alpha+\pi) / 3$, $T=6 \mathbf{a}$ and $T=8 \mathbf{b}$. As rotation angle approaches the critical rotation angles $\left(\alpha \rightarrow \alpha_{c}\right)$, the curvature function peaks and for $\alpha=\alpha_{c}$, the peak diverges and flips its signs. The critical rotation angles are $\alpha=0$ and $\pi / 4$. Since only one peak emerges in our diagrams, the critical behavior of the system can be described by peak-divergence scenario and band crossing is equal to one. If the gap-closer happens for $E=0$, the peak of curvature function is negative (positive) before (after) the critical point. The opposite is observed for if the energy of gap-closer is $E= \pm \pi$

$$
\lim _{\alpha \rightarrow 0^{-}} F\left(k_{c}, \alpha\right)=\lim _{\alpha \rightarrow \frac{\pi}{4}^{-}} F\left(k_{c}, \alpha\right)=\infty,
$$

Additionally, we can confirm that as rotation angles approach to critical rotation angle where energy gap closes $\left(\alpha \leftarrow \alpha_{c}\right)$, the curvature function starts to peak at gapless point in $k$ space parameter (see Fig. 2). The peak diverges when rotation angle becomes the critical rotation angle and flips its sign as it passes critical point. This is consistent with the results in Ref. [39]. Since the number of peaks is only one, the critical behavior of the system is peak-divergence type and the crossing band is one $(m=1)$. The curvature function has a Lorentzian shape. This indicates that we can use the Ornstein-Zernike formula to fit its peak

$$
F\left(k_{c}+\delta k, \alpha\right)=\frac{F\left(k_{c}, M\right)}{1 \pm \xi^{2} \delta k^{2}},
$$

where $\xi$ is length scale. The quantum walk simulates BDI family of topological phases, and this essentially means Su-Schrieffer-Heeger model. For $k=0$, the curvature function reduces to

$$
F(k=0, \alpha)=-\kappa_{\alpha} \csc \left(\frac{T(\alpha+\beta)}{2}\right)
$$

which consequently gives us the length scale as

$$
\begin{aligned}
\xi^{2}= & -\frac{1}{8} \kappa_{\beta} \csc ^{2}\left(\frac{T(\alpha+\beta)}{2}\right)\left[\sin \left(\frac{T(3 \alpha+2 \beta)}{2}\right)\right. \\
& \left.+3 \sin \left(\frac{T(\alpha+2 \beta)}{2}\right)+2 \lambda_{\alpha}\right] .
\end{aligned}
$$

The length scale and curvature function are both divergent for $\alpha=\alpha_{c}$. The critical exponents for this system are read as $\gamma=v=1$ since $F(k=0, \alpha) \propto\left|\alpha-\alpha_{c}\right|^{-1}$ and $\xi \propto\left|\alpha-\alpha_{c}\right|^{-1}$. This indicates that the scaling law will be given by $\gamma=v$. In the next step, we investigate the correlation function for our quantum walks. The correlation function is 
(a)

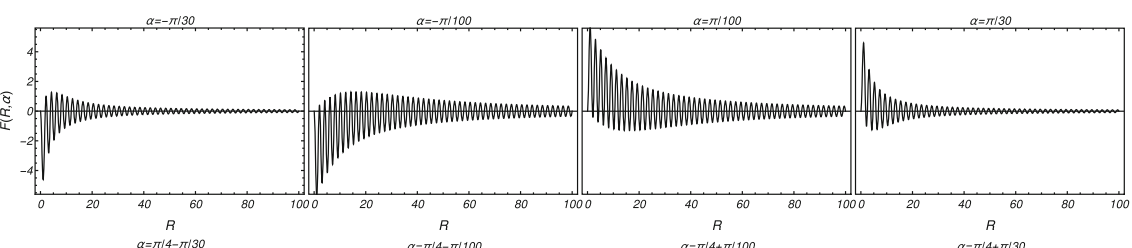

(b)

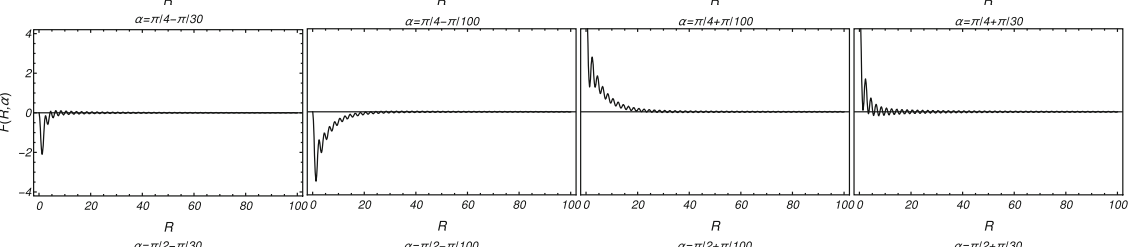

(c)

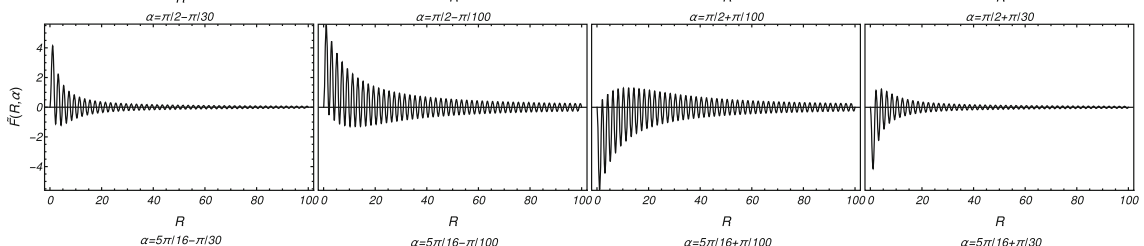

(d)

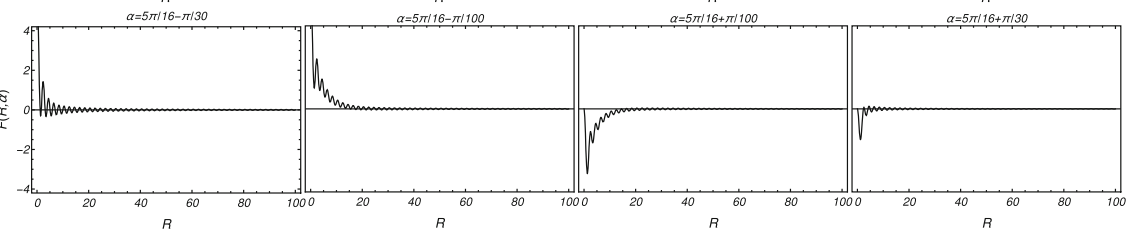

Fig. 3 Curvature function as a function of momentum for different rotation angle of $\alpha$ with $\beta=(\alpha+\pi) / 3$, $T=6 \mathbf{a}$ and $T=8 \mathbf{b}$. The correlation function decays as a function of $R$. The decay rate depends how close $\alpha$ is to critical point. The characteristic behavior of correlation function depends on type of boundary state; for Dirac cone boundary state, the decay happens through a strong and slower damped oscillation, while for Fermi arc boundary state, it happens faster. In addition, the correlation function for Dirac cone boundary state admits $\tilde{F}\left(R, \alpha_{c}-\alpha\right)=-\tilde{F}\left(R, \alpha_{c}+\alpha\right)$, while the same does not hold for Fermi arc boundary state

Fourier transformation of the curvature function using Wannier state which is given by

$$
\tilde{F}(R, \alpha)=\int_{0}^{2 \pi} \frac{d k}{2 \pi} F(k, \alpha) e^{i k R}
$$

in which we have used

$$
|\boldsymbol{R}\rangle=\frac{1}{N} \sum_{k} e^{i \boldsymbol{k}(\hat{r}-R)}\left|\psi_{\boldsymbol{k}-}\right\rangle,
$$

where $\left|\psi_{\boldsymbol{k}_{-}}\right\rangle$is the eigenstate of the Hamiltonian. Using Eq. (12), it is a matter of calculation to show that the correlation function decays through $\tilde{F}(R, \alpha) \propto e^{-\frac{R}{\xi}}$. The plotted diagrams for the correlation function confirm the decay (see Fig. 3). The type of the decay depends on the type of the boundary state. For Dirac cone boundary state, the decay happens through a strong damped oscillation and it happens rather slower comparing to Fermi arc boundary state. For Fermi arc boundary state, the decay happens faster and it is through non-damped oscillation. The presence of the damped oscillation is rooted in the fact that for Dirac cone boundary states, the curvature function forms additional peaks at $k= \pm \pi$ due to simultaneous gap-closer at $k=0$ and $\pm \pi$. In addition, the correlation function for Dirac cone boundary state admits $\tilde{F}\left(R, \alpha_{c}-\alpha\right)=-\tilde{F}\left(R, \alpha_{c}+\alpha\right)$ which is also evident from plotted diagrams in 
Fig. 3a and 3c. In contrast, such equality does not hold for correlation function of the Fermi arc boundary states (see Fig. 3b, d).

As final remarks, we should address phase transition points located at flat bands boundary states. The peak-divergence scenario and curvature function cannot be applied for investigating the critical behavior of the system with flat bands. At the heart of method using curvature function lies the dependency on the momentum, $k$. The flat bands in one-dimensional systems are independent of the $k$ [33]. Therefore, we cannot use this method to investigate critical behavior. All of the discussions that we have presented here for $T=6$ can be applied for $T=8$ and also in the case of position-dependent rotation angle. The results would be similar to those discussed for $T=6$ with consideration of modifications in gapless energy bands that would take place.

In conclusion, we showed that utilization of the step-dependent coins in quantum walk could result in formation of novel cell-like topological structures. These cell-like topological structures contain all possible boundary (edge) states alongside of all topological phases of BDI family. Due to dynamicality introduced by step-dependent coins, the cell-like structure would emerge in different steps. This enables us to use the step number as a mean to simulate desirable number of cell-like structures with optimized interior topological phases and boundary (edge) states. In addition, we used the Bloch sphere presentation of $\boldsymbol{n}(k)$ to highlight differences between different boundary states. Next, we used curvature function to investigate topological phase transitions and the critical behavior of the simulated topological phenomena. We confirmed that critical behavior of the system could be described by peak-divergence scenario. In addition, we showed that the band crossing for these critical behaviors is one. We calculated length scale, extracted critical exponents and validate the scaling law. Finally, we obtained the correlation function through Fourier transformation of Wannier states and showed that it decays as a function of the length scale. The characteristic behavior of decay for Fermi arc and Dirac cone boundary states was quite different due to the geometry of their gap-closer.

S.P would like to thank Andreas P. Schnyder and Wei Chen for helpful discussions.

Acknowledgements Open Access funding provided by Projekt DEAL.

Open Access This article is licensed under a Creative Commons Attribution 4.0 International License, which permits use, sharing, adaptation, distribution and reproduction in any medium or format, as long as you give appropriate credit to the original author(s) and the source, provide a link to the Creative Commons licence, and indicate if changes were made. The images or other third party material in this article are included in the article's Creative Commons licence, unless indicated otherwise in a credit line to the material. If material is not included in the article's Creative Commons licence and your intended use is not permitted by statutory regulation or exceeds the permitted use, you will need to obtain permission directly from the copyright holder. To view a copy of this licence, visit http://creativecommons.org/licenses/by/4.0/.

\section{References}

1. D.J. Thouless et al., Quantized hall conductance in a two-dimensional periodic potential. Phys. Rev. Lett. 49, 405 (1982)

2. X.L. Qi, T.L. Hughes, S.C. Zhang, Topological field theory of time-reversal invariant insulators. Phys. Rev. B 78, 195424 (2008)

3. M.Z. Hasan, C.L. Kane, Topological insulators. Rev. Mod. Phys. 82, 3045 (2010)

4. X.L. Qi, S.C. Zhang, Topological insulators and superconductors. Rev. Mod. Phys. 83, 1057 (2011)

5. L. Fidkowski, A. Kitaev, Topological phases of fermions in one dimension. Phys. Rev. B 83, 075103 (2011)

6. M. Koenig et al., Quantum spin hall insulator state in HgTe quantum wells. Science 318, 766 (2007) 
7. D. Hsieh et al., A topological Dirac insulator in a quantum spin Hall phase. Nature 452, 970 (2008)

8. M. Atala et al., Direct measurement of the Zak phase in topological Bloch bands. Nat. Phys. 9, 795 (2013)

9. M. Leder et al., Real-space imaging of a topologically protected edge state with ultracold atoms in an amplitude-chirped optical lattice. Nat. Commun. 7, 13112 (2016)

10. R. Süsstrunk, S.D. Huber, Observation of phononic helical edge states in a mechanical topological insulator. Science 349, 47 (2015)

11. T. Kitagawa et al., Observation of topologically protected bound states in photonic quantum walks. Nat. Commun. 3, 882 (2012)

12. F. Cardano et al., Statistical moments of quantum-walk dynamics reveal topological quantum transitions. Nat. Commun. 7, 11439 (2016)

13. K. Wang et al., Simulating dynamic quantum phase transitions in photonic quantum walks. Phys. Rev. Lett. 122, 020501 (2019)

14. F. Cardano et al., Detection of Zak phases and topological invariants in achiral quantum walk of twisted photons. Nat. Commun. 8, 15516 (2017)

15. S. Barkhofen et al., Measuring topological invariants in disordered discrete-time quantum walks. Phys. Rev. A 96, 033846 (2017)

16. E. Flurin et al., Observing topological invariants using quantum walks in superconducting circuits. Phys. Rev. X 7, 031023 (2017)

17. X. Zhan et al., Detecting topological invariants in nonunitary discrete-time quantum walks. Phys. Rev. Lett. 119, 130501 (2017)

18. L. Xiao et al., Higher winding number in a nonunitary photonic quantum walk. Phys. Rev. A 98, 063847 (2018)

19. N.B. Lovett et al., Universal quantum computation using the discrete-time quantum walk. Phys. Rev. A 81, $042330(2010)$

20. M. Mohseni et al., Environment-assisted quantum walks in photosynthetic energy transfer. J. Chem. Phys. 129, $174106(2008)$

21. I. Vakulchyk, M.V. Fistul, S. Flach, Wave packet spreading with disordered nonlinear discrete-time quantum walks. Phys. Rev. Lett. 122, 040501 (2019)

22. T. Kitagawa et al., Exploring topological phases with quantum walks. Phys. Rev. A 82, 033429 (2010)

23. T. Kitagawa, Topological phenomena in quantum walks: elementary introduction to the physics of topological phases. Quant. Info. Proc. 11, 1107 (2012)

24. J.K. Asboth, Symmetries, topological phases, and bound states in the one-dimensional quantum walk. Phys. Rev. B 86, 195414 (2012)

25. H. Obuse et al., Unveiling hidden topological phases of a one-dimensional Hadamard quantum walk. Phys. Rev. B 92, 045424 (2015)

26. T. Chen, B. Wang, X. Zhang, Characterization of topological phases and selection of topological interface modes in the parity-time-symmetric quantum walk. Phys. Rev. A 97, 052117 (2018)

27. S. Panahiyan and S. Fritzsche, Toward simulation of topological phenomenas with one-, two- and threedimensional quantum walks, [arXiv:2005.08720]

28. V.V. Ramasesh et al., Direct probe of topological invariants using bloch oscillating quantum walks. Phys. Rev. Lett. 118, 130501 (2017)

29. J.K. Asboth, Bulk-boundary correspondence for chiral symmetric quantum walks. Phys. Rev. B 88, 121406(R) (2013)

30. B. Tarasinski, J.K. Asboth, J.P. Dahlhaus, Scattering theory of topological phases in discrete-time quantum walks. Phys. Rev. A 89, 042327 (2014)

31. T. Rakovszky, J.K. Asboth, Localization, delocalization, and topological phase transitions in the onedimensional split-step quantum walk. Phys. Rev. A 92, 052311 (2015)

32. B. Mera et al., Boltzmann-Gibbs states in topological quantum walks and associated many-body systems: fidelity and Uhlmann parallel transport analysis of phase transitions. J. Phys. A: Math. Theor. 50, 365302 (2017)

33. S. Panahiyan and S. Fritzsche, Controllable simulation of topological phases and edge states with quantum walk, [arXiv:2004.04042]

34. S. Panahiyan, S. Fritzsche, Controlling quantum random walk with a step-dependent coin. New J. Phys. 20, 083028 (2018)

35. C.K. Chiu et al., Classification of topological quantum matter with symmetries. Rev. Modern Phys. 88, 035005 (2016)

36. J.K. Asboth, L. Oroszl, A. Palyi, A Short Course on Topological Insulators (Springer, Berlin, 2016)

37. C. Yin et al., Geometrical meaning of winding number and its characterization of topological phases in one-dimensional chiral non-Hermitian systems. Phys. Rev. A 97, 052115 (2018) 
38. L. Li, C. Yang, S. Chen, Winding numbers of phase transition points for one-dimensional topological systems. Euro. Phys. Letts. 112, 10004 (2015)

39. W. Chen, A.P. Schnyder, Universality classes of topological phase transitions with higher-order band crossing. New J. Phys. 21, 073003 (2019)

40. W. Chen et al., Correlation length, universality classes, and scaling laws associated with topological phase transitions. Phys. Rev. B 95, 075116 (2017) 